Furthermore, colleagues who have failed fittesting due to their anatomy may also be faced with a similar predicament where the local trust is unable to provide a respirator hood.

Clinical activities for such colleagues will be limited to non-aerosol generating procedures. For colleagues in surgical training pathways, eg dental core training or middlegrade staff, this presents a further frustration: the specialty job application for oral surgery may ask how many surgical procedures they have carried out, and assign a score based on this. Colleagues who find themselves in the aforementioned predicaments may be disadvantaged. Should this element of the application process be revised?

I. Rehman, Glasgow, UK

https://doi.org/10.1038/s41415-020-2459-9

\section{PPE consistency please}

Sir, with regard to protection, since the beginning of the pandemic the UK Government has changed its Infection prevention and control guidance 37 times. ${ }^{1}$ This easily leads to confusion within hospitals and can often result in individuals following their personal interpretation of the guidance.

We would like to highlight the need for ongoing vigilance and consistency by all members of the team. An example within our hospital operating theatre was noted where five clinicians, including anaesthetists, oral surgeons, nurses and students all wore differing levels and styles of personal protective equipment (PPE) whilst working on the same case. Ideally, PPE recommendations for each case should be covered at the team brief at the start of a list and repeated during the WHO checklist. Clarity from the start of the case is paramount to effective safe operating and reassures all personnel that they are adequately protected with the chosen PPE.

The Royal College of Surgeons stated online that members should not put themselves at risk due to inadequate PPE whilst also stating that the guidance to be followed should be based on each individual trust protocol. ${ }^{2}$ This also leads to discrepancies with trainees often rotating around various hospitals. It now appears likely that managing the risk of COVID-19 will be a challenge for clinicians for the foreseeable future. We believe there is a need for consistency and ongoing education regarding PPE both at a national and local level to ensure safety of all members of the clinical team and patients.

E. Thomas, D. Cunliffe, Torbay; O. Rees-Stoner, B. Collard, Plymouth, UK

\section{References}

1. Public Health England. COVID-19: infection prevention and control (IPC). London, 2020. Available at: https://www.gov. uk/government/publications/wuhan-novel-coronavirusinfection-prevention-and-control\#history (accessed 21 October 2020).

2. Royal College of Surgeons. Don't Risk It! London, 2020. Available at: https://www.rcseng.ac.uk/news-and-events/ blog/coronavirus-ppe-dont-risk-it/ (accessed 21 October 2020).

https://doi.org/10.1038/s41415-020-2460-3

\section{Failure to attend or fear to attend?}

Sir, I write from a district general hospital, reflecting on the rise in patients failing to attend appointments. Could it be that with increased awareness of COVID-19, patients have again become fearful of the risks associated with attending hospitals?

Where does the line get drawn between what can wait and what is necessary? Can a symptomatic tooth be left untreated until a severe abscess or swelling develops, requiring hospital admission? Can a two-week wait appointment go missed and a lesion only be investigated at the point it is more advanced and complex to treat? As clinicians it is far simpler to answer these questions, but we cannot expect the same difficult decisions to be made by our patients who are looking to us for advice in these times.

Recently, when questioning a patient presenting with a carcinoma of the lip on why they left this un-investigated for many months, the response we have become accustomed to hearing was given, 'COVID'. We want to encourage patients to feel comfortable attending their appointments, but how do we do this in a way that doesn't disregard the rising number of cases? What we can do is offer clear advice to our patients about precautions being implemented in healthcare settings, and what the patient themselves can do before the appointment. With the majority of complaints stemming from poor communication, it is important that the message we send to patients is clear, informative and consistent, and one that does not instil fear but instead inspires confidence. $^{2}$

L. Smith, Torbay, UK

\section{References}

1. Giles C. Ceinwen Giles: Is it safe to go to hospital or visit my GP-patients want to know. 18 September 2020. Available at: https://blogs.bmj.com/bmj/2020/09/18/ceinwengiles-is-it-safe-to-go-to-hospital-or-visit-my-gp-patientswant-to-know/ (accessed November 2020).

2. Abdelrahman $W$, Abdelmageed A. Understanding patient complaints. BMJ 2017; 356: DOI: https://doi.org/10.1136/ bmj.j452.

https://doi.org/10.1038/s41415-020-2461-2

\section{Mental health}

\section{Compromised wellbeing}

Sir, my clinical supervisor shared with us earlier today that one of her patients broke down in tears in two consecutive appointments, due to major changes in the patient's life impacted by the COVID-19 pandemic. I learnt that the negative impact of the COVID-19 pandemic on mental health can be a lot more prevalent than we all like to think.

Undoubtedly, the impact of the COVID-19 pandemic is extensive on people's lives. It is possible that every one of us knows someone that has, or we ourselves have, suffered from bereavement, financial or unemployment worries, parenting challenges, domestic conflicts, a sense of loneliness, fear of uncertainty, or any other stressors that can compromise our mental wellbeing, at some point during the outbreak.

However, signs and symptoms of compromised mental wellbeing can be difficult to pick up under social distancing measures. As dental practitioners, we have the opportunity to meet colleagues and patients for face-to-face treatments, which put us in a unique position to recognise mental health problems. But the question is, where should we signpost them to?
Before referring someone to their GP or the NHS mental health service, remember, sometimes, all the individual needs is a good listener. It is therefore important to have appropriate information on alternative resources available and signpost accordingly. Specific tips and advice on different challenges faced during the COVID-19 pandemic can be accessed at https://www.mentalhealth.org. $\mathrm{uk}$; a list of NHS-recommended helplines is available at https://www.nhs.uk/conditions/ stress-anxiety-depression/mental-healthhelplines/; and local helplines and mental health organisations should also be explored and utilised. 